\title{
Short wavelength automated perimetry in age related maculopathy
}

\author{
Andreas Remky, Kristina Lichtenberg, Ann E Elsner, Oliver Arend
}

\begin{abstract}
Backgroundlaims-Previous studies reported the predictive value of the short wavelength sensitive (SWS) cone mediated sensitivity for visual outcome in age related macular degeneration. In this study SWS sensitivity was measured by commercially available blue on yellow perimetry in patients with non-exudative age related maculopathy (ARM) and compared with the presence of morphological risk factors and the status of the fellow eye.
\end{abstract}

Methods-In a prospective cross sectional study, 126 patients (57 males, 69 females, mean age 71 (SD 6) years) with ARM (visual acuity $>20 / 50$ ) were tested. Central visual fields (blue on yellow) were obtained with a conventional perimeter. Fundus slides were graded by two independent observers for soft drusen and presence of focal hyperpigmentation.

Results-Mean sensitivity and standard deviation of all patients exhibited a significant reduction with age. Patients with soft drusen had significantly lower sensitivity than those without, whereas there were no differences in visual acuity (log MAR). Sensitivity was also reduced in those eyes with fellow eyes having a sight threatening complication of age related macular degeneration (AMD). Eyes with focal hyperpigmentation compared with those without had no loss of sensitivity, but did have a significant decrease in the central part of the field compared with the more eccentric.

Conclusion-SWS sensitivity loss is associated with common risk factors for progression to AMD. Short wavelength automated perimetry is moderately rapid and readily available. It may serve as a tool in future ARM trials.

(Br f Ophthalmol 2001;85:1432-1436)

Numerous studies ${ }^{1-7}$ have been performed to identify the risk factors for age related macular degeneration (AMD), the most common cause for legal blindness in industrialised countries. ${ }^{89}$ The more severe forms of AMD include geographic atrophy, detachments of the retinal pigment epithelium, and/or new vessel growth between Bruch's membrane and the retina. Since effective laser treatment is available only for certain exudative cases, primarily in the early stages of a rapidly changing process, ${ }^{10}$ the establishment of risk factors for an exudative outcome is crucial. An accurate estimate for retinal health outside the central fovea may be mandatory for modern surgical procedures such as macular rotation and transplantation of pigment epithelium, ${ }^{11}{ }^{12}$ and advanced therapeutic treatments such as photodynamic therapy. ${ }^{13}$

Early fundus changes in AMD, such as drusen, small areas of atrophy, and hyperpigmentation are visible in a high percentage of the elderly population. ${ }^{14}{ }^{15}$ Evidence of any of these fundus features is now called age related maculopathy (ARM), as distinguished from the more advanced stages of degeneration called age related macular degeneration (AMD) ${ }^{16}$ Although some aspects of ARM, including type and amount of pathology, have been shown to have prognostic value, ${ }^{617}{ }^{18}$ the predictive value of morphological factors is low, possibly since the incidence of age related fundus changes is high. ${ }^{15}$

Several recent reports have shown the advantages of short wavelength automated perimetry (SWAP) for indicating early damage in patients with ocular hypertension and glaucoma. ${ }^{19-21}$ The principle of SWAP is the isolation of the short wavelength sensitive (SWS) cone response by suppression of the responses of the other cone types by using a yellow background. ${ }^{22} 23$ The method is established in the early detection of glaucoma, ${ }^{21} 24$ where its use is to detect changes predominantly at the retinal ganglion cell level and loss of retinal nerve fibres. SWS mechanisms are also reported to be susceptible to damage in a variety of retinal diseases, in which changes are less specific for retinal nerve fibres and probably related to other alterations of the inner retina. ${ }^{25-28}$

In this study, we employed short wavelength automated perimetry (SWAP) in the 10 degree central field in a large sample of patients with ARM. In contrast with previous reports of SWS sensitivity measurements, a standard perimeter $^{29}$ was used which is familiar to most clinicians, and equipped with a commercially available modification. SWS cone sensitivity was compared to the presence of established risk factors such as drusen, focal hyperpigmentation, and the status of the second eye.

\section{Material and methods}

PATIENTS

Patients with ARM and acuity of better than $20 / 50$ were recruited in a prospective study. The diagnosis was based on presence of any drusen, hyperpigmentation, and/or small atrophic lesions. Eyes with any atrophic area greater than $200 \mu \mathrm{m}$ in diameter on clinical examination, any exudative lesions, or any history of eye disease that may have impact on retinal function were excluded. In $85 \%$ of patients combined fluorescein/indocyanine 
green angiography was performed to rule out any early or occult exudative lesion. The eye with the better visual acuity was selected; in case of equal acuity, the eye with the lower refractive error was chosen. Slit lamp examination of the anterior segment was normal in all eyes. Nuclear sclerosis was graded with retroilluminated match templates (LOCS III) in five categories (NC 0-4)..$^{30}$ Eyes with moderate and advanced nuclear opacities (NC 3 and NC 4) were excluded.

Best corrected visual acuity was determined by an ophthalmologist using objective refractometry (Rodenstock, Germany), the lighting conditions and the standardised charts described by DIN $58220,{ }^{31}$ followed by a complete ophthalmological examination. For statistical analysis all visual acuity scores were converted into logarithmic equivalents (logMAR).

Informed consent was obtained from each subject including detailed explanations of all procedures before participation in this study. The protocol for the study was reviewed and approved by the RWTH University institutional review board for the use of human subjects.

\section{METHODS}

A Humphrey visual field analyser (Model 750, Humphrey-Zeiss, San Leandro, CA, USA) was used for SWAP, as described in described in recent reports. ${ }^{32}$ The stimulus size was Goldmann $\mathrm{V}$ (1.8 degree visual angle). The background was a $100 \mathrm{~cd} / \mathrm{m}^{2}$ yellow. A full threshold strategy was applied for the central 10 degree field (Program 10-2). Figure 1 shows the test pattern projected on a fundus photograph in a patient with soft drusen. Patients with more than $20 \%$ false positive or false negative responses were excluded.

Global indices (mean defect, pattern standard deviation, or short term fluctuation) are not available for SWAP 10 degree fields, owing to the lack of normative data. Data analysis was derived from all 68 single data points in digital work sheets. Data entry was performed twice by different operators to minimise errors. For

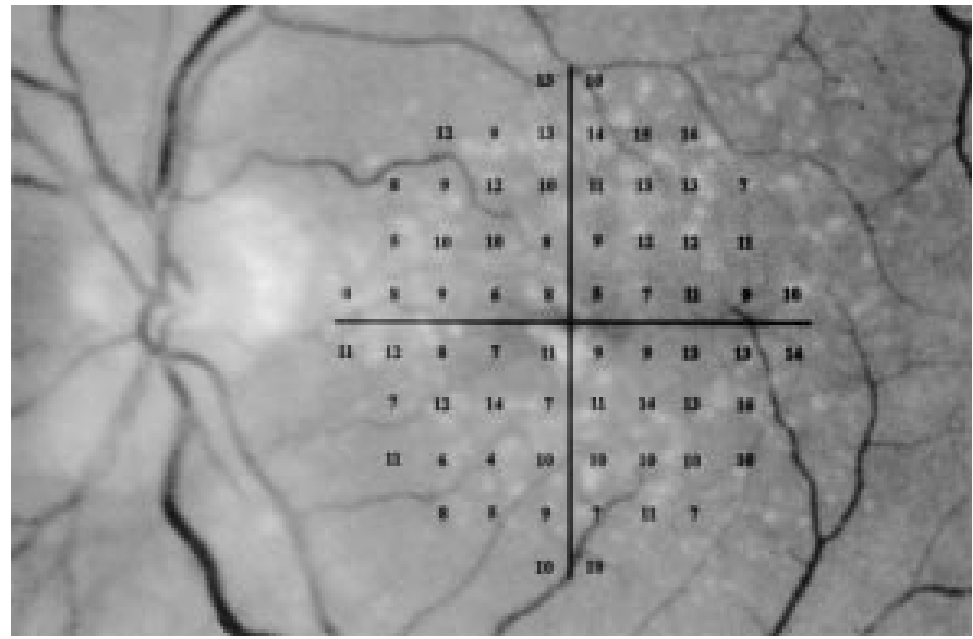

Figure 1 Perimetry results (in $d B$ ) for all 68 stimulus locations superimposed on the matching fundus photograph. A 68 year old male patient with normal visual acuity and soft drusen. the analysis of overall function, the mean sensitivity was calculated. For sensitivity changes across the field, the standard deviation of all 68 locations was used. For the analysis of regional differences, means were calculated for locations within the central 5 degree field $(n=24)$ and the more eccentric locations $(n=44)$.

After dilatation and funduscopy, photography was performed to assess 30 degree macula centred colour slides. These slides were graded by two independent observers ( $\mathrm{AR}$ and $\mathrm{OA}$ ) who were masked as to the identity of patients and perimetry results. A template with a 10 degree circle centred on the fovea was superimposed on a slide viewer. Drusen were assigned to one of two categories: hard or soft. ${ }^{1633}$ Soft drusen were classified as having ill defined borders, with a diameter of greater than $63 \mu \mathrm{m}$. The macula was graded in the 10 degree field for absence or presence of soft drusen, focal hyperpigmentation, and hard drusen which were defined as rounder lesions with discrete borders and a size smaller than 63 $\mu \mathrm{m}$. The interobserver agreement was $90 \%$. Twelve slides were discussed until agreement was reached. In the grading process, one eye with chronic central serous chorioretinopathy and three eyes with atrophic lesions greater than $200 \mu \mathrm{m}$ were excluded, for a total of 130 subjects before evaluation of the sensitivity data.

\section{STATISTICAL ANALYSIS}

Statistical analysis was performed using analysis of variance (ANOVA) as computed with STATVIEW (SAS Institute Inc, Cary, NC, USA). We investigated the relation of risk factors (age, status of second eye, presence of focal hyperpigmentation or soft drusen) to mean SWS sensitivity, standard deviation, and visual acuity (logMAR). A repeated measures ANOVA was used for testing the interaction of regional field differences and risk factors on mean SWS sensitivity.

For post hoc testing calculations with Fisher's protected least significant difference were carried out. Findings of a probability of a type error less than 0.05 were considered to be statistically significant. For the comparison of discrete variables, a $\chi^{2}$ test was calculated for each contingency table.

\section{Results}

One hundred and twenty six patients passed all screening criteria and were recruited, ranging in age from 55 to 87 years (mean age 71 (SD 6) years). Of these, 75 patients $(60 \%)$ had a sight threatening complication in the fellow eye. Three had vascular disease processes (central retinal vein occlusion, anterior optic neuropathy, and vitreomacular traction) in the fellow eye without evidence of atrophy or exudation due to AMD. Soft drusen were present in 93 patients $(74 \%)$, focal hyperpigmentation in 64 patients $(51 \%)$.

Mean SWS sensitivity (average of 68 locations), as a measure of overall function, decreased significantly (Table 1) with increasing age (Fig 2). There was no effect or interaction of sex on sensitivity. The standard 
Table 1 ANOVA table for the mean sensitivity of mean sensitivity. Owing to the large first order effect of age only the effect of soft drusen on sensitivity remained significant. $p$ Values of the post hoc tests are given in Table 2

\begin{tabular}{lcc}
\hline Factor & F value & $p$ Value \\
\hline Soft drusen & 4.34 & 0.039 \\
Hyperpigmentation & 0.49 & 0.487 \\
AMD of the fellow eye & 1.07 & 0.303 \\
Age & 23.0 & $<0.0001$ \\
\hline
\end{tabular}

deviation significantly increased with age ( $\mathrm{p}$ $<0.016$, ANOVA).

Figure 1 shows a typical example for a patient with soft drusen. Patients with soft drusen had significantly reduced mean sensitivity (Table 2) compared to those without. Few eyes without soft drusen had a significant reduction of sensitivity - for example, if an arbitrary cut-off value of $17 \mathrm{~dB}$ were chosen, two of $33(6 \%)$ eyes without soft drusen had mean sensitivity less than this value compared with 26 of $93(28 \%)$ eyes with soft drusen $(\mathrm{p}=$ 0.0077; Fig 2).

The difference in mean sensitivity was not due solely to the age effect, since patients with and without soft drusen did not differ in age and a significant main effect for soft drusen was found as well as for age in the ANOVA (Tables 1 and 2). The standard deviation across all 68 test loci was not significantly different in the eyes with drusen compared with those without (Table 2, Fig 2B). There was no significant difference in age and visual acuity expressed in $\log$ MAR between the eyes with and without soft drusen.

Mean sensitivity had a significantly steeper decline with age for patients with soft drusen than those without, as indicated by the slope test of the linear regression $(\mathrm{t}(91)=2.40$, one tailed test, $p<0.01)$ ). For the patients without soft drusen, the slope of the regression line for sensitivity as a function of age was not significantly different from 0 , given our sample size (one tailed test $=1.66, \mathrm{p}>0.05)$. For patients with soft drusen, the slope was significantly more negative than the slope of patients without soft drusen, and therefore also significantly less than 0 -that is, there was a significant decline in sensitivity with age only for patients with soft drusen. This implies that while there might be a slight decrease of sensitivity due to ageing factors unrelated to the underlying processes that cause soft drusen, this decrease is far less than the result of the combination of ageing processes and the processes leading to soft drusen. As our patients had only mild or moderate lens changes, then the lack of a significant trend with age for
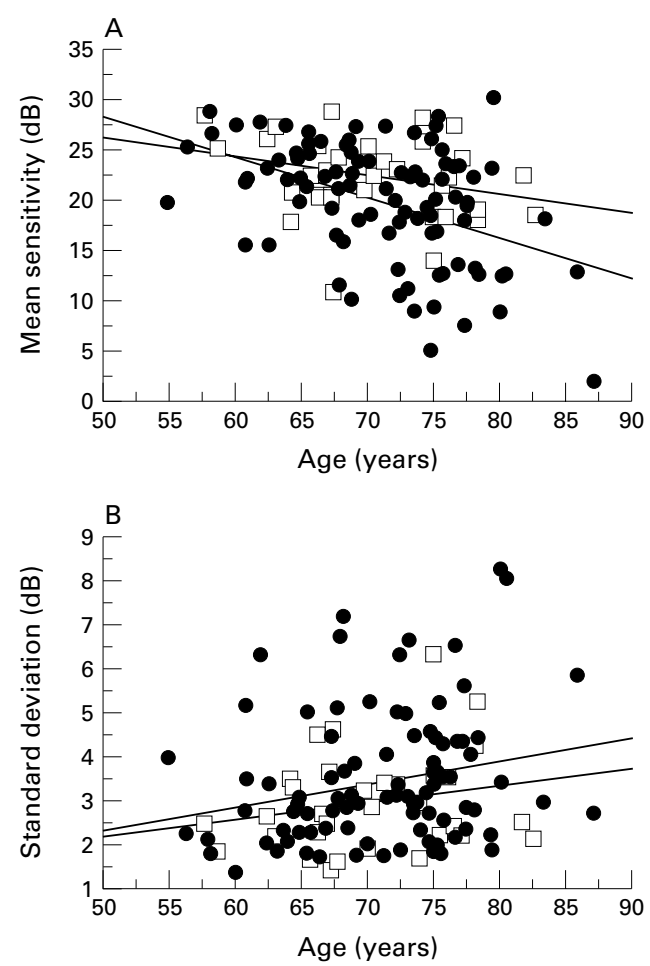

Figure 2 (A) Mean sensitivity of the included patients as a function of age (mean sensitivity $=45.77-0.357 \star$ age; $r$ $=-0.41$ ); closed circles: eyes with soft drusen (mean sensitivity $=48.41-0.403 *$ age; $r=-0.45)$, open squares: eyes without soft drusen (mean sensitivity $=35.67-0.190$ * age; $r=-0.29$ ). (B) Standard deviation (SD) over the full 10 deg field of the included patients as a function of age $(S D=-0.283+0.051 *$ age; $r=0.23)$; closed circles: eyes with soft drusen $(S D=-0.335+0.053 \star$ age $r=0.23)$, open squares: eyes without soft drusen $(S D=0.273+$ 0.039 *age $; r=0.22$ ).

patients without soft drusen could be expected. This confirms that our patient selection criteria, including cataract screening, were sufficient to result in ageing data that revealed differences in sensitivity with fundus changes and not only with the media changes that are prevalent with ageing.

The study sample included 75 fellow eyes of an exudative or severe atrophic AMD. The incidence of soft drusen was not significantly different between these two groups, but hyperpigmentation was observed more frequently $\left(\chi^{2}\right.$, presence of soft drusen: $\mathrm{p}=0.07$, presence of hyperpigmentation: $p=0.006$ ). Mean SWS sensitivity was significantly reduced in fellow eyes of patients with severe AMD compared to those without (Table 2). This effect was not significant in the ANOVA when the age factor was simultaneously analysed (Table 1), consistent with age distribution in Figure 2 that shows

Table 2 Demographic and perimetric data of the eyes with and without the tested factors. $p$ Values are calculated with the Fisher's PLSD test

\begin{tabular}{|c|c|c|c|c|c|c|c|c|c|c|}
\hline & \multicolumn{3}{|l|}{ Soft drusen } & \multicolumn{3}{|c|}{ Focal hyperpigmentation } & \multicolumn{4}{|c|}{ Status of fellow eye } \\
\hline & Present & Absent & $p$ Value & Present & Absent & $p$ Value & $A R M$ & & $A M D$ & $p$ Value \\
\hline No & 93 & 33 & & 64 & 62 & & 51 & & 75 & \\
\hline Visual acuity & 0.75 & 0.82 & & 0.75 & 0.80 & & 0.8 & & 0.75 & \\
\hline $\log$ MAR $(\mathrm{SD})^{\star}$ & $0.12(0.12)$ & $0.08(0.11)$ & 0.065 & $0.13(0.12)$ & $0.10(0.11)$ & 0.17 & 0.0 & $(0.12)$ & $0.13(0.12)$ & 0.13 \\
\hline Age (years) (SD) & $71 \quad(6)$ & $70 \quad(6)$ & 0.64 & $71 \quad(6)$ & $71 \quad(7)$ & 0.56 & & (7) & $72 \quad(6)$ & 0.06 \\
\hline Mean sensitivity (dB) (SD) & 19.8 & $22.3(4.1)$ & 0.027 & 20.0 & 21.0 & 0.31 & 21.6 & $(5.2)$ & $19.7(5.7)$ & 0.049 \\
\hline SD of sensitivity $(\mathrm{dB})$ & $3.5(1.5)$ & $3.0(1.1)$ & 0.10 & $3.6(1.5)$ & $3.3(1.4)$ & 0.064 & 3.1 & (1.3) & $3.5(1.5)$ & 0.17 \\
\hline
\end{tabular}

^Standard deviation. 
many older patients with the poorest sensitivity had soft drusen.

Eyes with focal hyperpigmentation did not differ significantly in either age or visual acuity, compared to eyes without. Mean sensitivity and standard deviation were not statistically related to the presence of focal hyperpigmentation (Table 2). To evaluate the eccentricity effects, an additional analysis of the more central versus the more eccentric test points was performed. There was no significant difference in the average sensitivity between the inner 24 points within the central 5 degree versus the other 42 points outside for the full sample. The presence of hyperpigmentation, however, was related to a significant eccentricity effect (ANOVA: $p=0.0006)$. Eyes with focal hyperpigmentation had a central decrease of 0.67 $\mathrm{dB}$, eyes without hyperpigmentation an increase of $0.73 \mathrm{~dB}$ compared with the more eccentric average sensitivity. All other factors had no impact on eccentricity effects.

\section{Discussion}

In this study a Humphrey perimeter, an item of standard equipment in many clinics and practices, was used to assess SWS cone mediated sensitivity. Previous promising methods to measure foveal SWS sensitivity failed to be adopted clinically owing to instrumental or other constraints. A significant decrease of sensitivity was detected in eyes with risk factors for AMD such as age, the presence of soft drusen, and fellow eyes already having AMD. The effect of focal hyperpigmentation on sensitivity was only significant for the central 5 degree field.

The strong relation of sensitivity to age is not surprising, since age is the major risk factor in AMD. The decrease in sensitivity with age cannot be attributed to the effects of preretinal media absorption alone. There was no significant difference in age for patients with as opposed to without soft drusen in our sample. Yet there was no significant age dependency of sensitivity in the subgroup without soft drusen, while there was significant age dependency for the subgroup with soft drusen (Fig 2). In addition, the average difference in sensitivity between patients with and without soft drusen was $2.5 \mathrm{~dB}$, despite the lack of difference in age.

The patients included in the study had no or moderate loss of visual acuity. There were no significant effects on visual acuity (expressed in $\log$ MAR) of the risk factors examined among patients with $20 / 25$ or better acuity. This is in accordance with other studies concerning functional abnormalities in ARM indicating poor prognostic value in AMD. ${ }^{35}{ }^{36}$ This study indicated that a loss of SWS cone mediated sensitivity might act as a functional risk factor as suggested by previous reports. ${ }^{35}{ }^{36}$ Several studies have previously shown functional abnormalities - for example, decreased retinal sensitivity under dark adapted ${ }^{37-39}$ or SWS cone mediated sensitivity, ${ }^{17}$ in patients with drusen and hyperpigmentation. Furthermore, the change of SWS sensitivity over time correlates well with changes of fundus appearance. ${ }^{35}{ }^{40} \mathrm{In}$ a longitudinal study Eisner et $a l^{36}$ found an association of low foveal S cone sensitivity with an exudative outcome. Foveal SWS cone testing in Maxwellian view, however, has not achieved widespread clinical use.

In early glaucoma short wavelength sensitivity is considered to be related to ganglion cell loss ${ }^{20}$ in diabetic retinopathy to compromised function of other retinal tissues. ${ }^{27}{ }^{28}$ In ARM, as a disease of the photoreceptor/retinal pigment epithelial (RPE) complex, ${ }^{14}{ }^{41}{ }^{42}$ the nature of the disease is different from the above disease processes, and the site of the sensitivity loss might be the outer retina or the photoreceptors themselves.

The global loss of sensitivity in eyes with soft drusen reflects the diffuse character of the disease. In contrast, the presence of focal hyperpigmentation, which was always graded centrally, resulted only in a sensitivity loss in the central 5 degrees without a significant reduction of the mean sensitivity of the entire central field. The method covering the entire 10 degrees may provide additional information outside the fovea-for example, localised losses. ${ }^{43}$ In patients already presenting with atrophy, scars, or neovascularisation this might be helpful for visual rehabilitation or studies concerning improving rehabilitation-for example, patients with a more generalised loss may be poor candidates for rehabilitation efforts.

Assessment of short wavelength sensitivity with a conventional perimeter is a quick, non-invasive diagnostic tool and may be performed with a conventional perimeter that is already available at many locations worldwide. Since morphological risk factors have a high prevalence in the elderly population, functional measures gain more and more importance. Measurements of SWS sensitivity may lead to new practical means of assessing ARM progression or treatment outcomes and should be considered for future ARM trials. Follow up studies to prove the prognostic value are in progress. Eisner A, Fleming SA, Klein ML, et al. Sensitivities in older human eyes with good acuity: eyes whose fellow eye has exudative AMD. Invest Ophthalmol Vis Sci 1987;28:1832-7.

2 Klein R, Klein BE, Jensen SC. The relation of cardiovasage-related mand its risk factors to the 5-year incidence of thalmology 1997;104:1804-12.

3 Laatikainen L, Hirvelae H. Prevalence and visual consequences of macular changes in a population aged 70 years and older. Acta Ophthalmol Scand 1995;73:105-10.

4 Macular Photocoagulation Study G. Risk factors for choroidal neovascularization in the second eye of patients with juxtafoveal or subfoveal choroidal neovascularization secondary to age-related macular degeneration. Arch Ophthalondary to age-related
mol 1997;115:741-7.

5 Midena E, Degli Angeli D, Blarzino MC, et al. Macular function impairment in eyes with early age-related macular degeneration. Invest Ophthalmol Vis Sci 1997;38:469-77.

6 Smiddy WE, Fine SL. Prognosis of patients with bilateral macular drusen. Ophthalmology 1984;91:271.

7 Vinding T, Appleyard M, Nyboe J, et al. Risk factor analysis for atrophic and exudative age related macular degeneration. Acta Ophthalmol Scand 1992;70:66-72.

8 Krumpaszky HG, Klau $\beta$ V. Epidemiology of blindness and eye disease. Ophthalmologica 1996;210:1-84.

9 Leibowitz H, Kruger DE, Maunder LR, et al. The Framingham Eye study Monograph: an ophthalmological and epidemiological study of cataract, glaucoma, diabetic retindemiological study of cataract, glaucoma, diabetic retinopathy, macular degeneration and visual acuity in a general population of 2631 adults,

10 Macular Photocoagulation Study Group. Laser photocoagulation for iuxtafoveal choroidal neovascularization. Arch Ophthalmol 1994;112:500-9. 
11 Rezai KA, Kohen L, Wiedemann P, et al. Iris pigment epithelium transplantation. Graefes Arch Clin Exp Ophthalepithelium transplanta
mol $1997 ; 235: 558-62$.

12 Wolf S, Lappas A, Weinberger AW, et al. Macular translocation for surgical management of subfoveal choroida neovascularization in patients with AMD. Graefes Arch Clin Exp Ophthalmol 1999;237:51-7.

13 Treatment of age-related macular degeneration with photodynamic therapy (TAP) study group Photodynamic therapy of subfoveal choroidal neovascularization in age-related macular degeneration with verteporfin: oneyear results of 2 randomized clinical trials-TAP report. Arch Ophthalmol 1999;117:1329-45.

14 Pauleikhoff D, Harper CA, Marshall J, et al. Aging changes in Bruch's membrane. Ophthalmology 1990;97:171-8.

15 Vingerling JR, Dielemans I, Hofman A, et al. The prevalence of age-related maculopathy in the Rotterdam Study. Ophthalmology 1995;102:205-10.

16 The International ARM Epidemiological Study Group. An international classification and grading system for ageinternational classification and grading system for agerelated maculopathy and age-related
tion. Surv Ophthalmol 1995;39:367-74.

17 Eisner A, Stoumbos VD, Klein ML, et al. Relations between fundus appearance and function. Invest Ophthalmol Vis Sci 1991;32:8-20.

18 Sarks SH. Drusen and their relationship to senile macular degeneration. Aust $\mathcal{F}$ Ophthalmol 1980;89:117-30.

19 Johnson CA, Adams AJ, Casson EJ, et al. Blue-on-yellow perimetry can predict the development of glaucomatous visual field loss. Arch Ophthalmol 1993;111:645-50.

20 Johnson CA, Adams AJ, Casson EJ, et al. Progression of early glaucomatous visual field loss as detected by blue-onyellow and standard white-on-white automated perimetry. Arch Ophthalmol 1993;111:651-6.

21 Sample PA, Martinez GA, Weinreb RN. Short-wavelength automated perimetry without lens density testing. $A m \mathcal{F}$ Ophthalmol 1994;118:632-41.

22 Johnson CA, Adams AJ, Twelker JD, et al. Age-related changes in the central visual field for short-wavelengthchanges in the central visual field for short-wave

23 Marré M, Marré E. Erworbene Störungen des Farbensehens. Diagnostik. Stuttgart: Gustav Fischer Verlag, 1986.

24 Johnson CA, Brandt JD, Khong AM, et al. Short wavelength sensitivity losses in low, moderate and high risk ocula hypertensives: initial baseline results in 250 Subjects. OSA Annual Meeting. 1994 Technical digest series 1994;16:290-3.

25 Crognale MA, Rabin J, Switkes E, et al. Selective loss of S-pathway sensitivity in central serous choroidopathy revealed by spatio-chromatic visual evoked cortical potential (VECP). In: Drum B, ed. Colour vision deficiencies XI. Amsterdam: Kluwer Academic Publishers, 1993;229-39.

26 Greenstein VC, Hood DC, Ritch R, et al. S (blue) cone pathway vulnerability in retinitis pigmentosa, diabetes and glaucoma. Invest Ophthalmol Vis Sci 1989;30:1732-7.

27 Hudson C, Flanagan JG, Turner GS, et al. Shortwavelength sensitive visual field loss in patients with clinically significant diabetic macular oedema. Diabetologia 1998;41:918-28.
28 Remky A, Arend O, Hendricks S. Short-wavelength automated perimetry and capillary density in early diabetic maculopathy. Invest Ophthalmol Vis Sci 2000;41:274-82

29 Mills RP, Hopp RH, Drance SM. Comparison of quantitative testing with the Octopus, Humphrey, and Tübingen Perimeters. Am f Ophthalmol 1986;102:496-504.

30 Siik S, Chylack LTj, Friend J, et al. Lens autofluorescence and light scatter in relation to the lens opacities classification systems, LOCS III. Acta Ophthalmol 1999;77.

31 Normenausschuß Feinmechanik und Optik:DIN 58220 (1974-1990), Bl. 3 (1974) Sehschärfenbestimmung, Teil 1 (1988) Normsehzeichen, Teil 2 (1989) Anschlu $\beta$ von Sehzeichen, Teil 3 (1990) Prüfung für Gutachten, Teil 4 (1980) Nahvisusbestimmung, Teil 5 (1986) Allgemeine Sehtestung, Teil 6 (1988) Straßenverkehrsbezogener Sehtest. In: Dtsch Institut für Normung eV Beuth B, ed. 1990.

32 Johnson CA, Brandt JD, Khong AM, et al. Short-wavelength automated perimetry in low-, medium-, and high-risk ocular hypertensive eyes. Arch Ophthalmol 1995;113:70-6.

33 Bressler NM, Bressler SB, Seddon JM, et al. Drusen characteristics in patients with exudative versus non-exudative age-related macular degeneration. Retina 1988;8:109-14.

34 Klein R, Davis MD, Magli YL, et al. The Wisconsin age-related maculopathy grading system. Ophthalmology 1991;98:1128-34.

35 Eisner A. Longitudinal changes of visual function over 18 months. Evaluation of eyes with high-and low-risk macular degeneration characteristics. In:Drum B, ed. Colour vision deficiencies XI. Amsterdam: Kluwer Academic Publishers, 1993:175-87.

36 Eisner A, Klein ML, Zilis JD, et al. Visual function and the subsequent development of exudative age-related macular degeneration. Invest Ophthalmol Vis Sci 1992;33:3091-102.

37 Brown B, Adams AJ, Coletta NJ, et al. Dark adaptation in age-related maculopathy. Ophthal Physiol Opt 1985;6:81-4.

38 Elsner AE, Burns SA, Weiter JJ. Cone photopigment in older subjects: dereased optical density in early age-related macular degeneration. $\mathcal{f} \mathrm{Opt} \operatorname{Soc} \mathrm{Am} A$ [in press].

39 Sunness JS, Massof RW, Johnson MA, et al. Diminished foveal sensitivity may predict the development of advanced age-related macular degeneration. Ophthalmology 1989;96: $357-81$.

40 Holz FG, Gross-Jendroska M, Eckstein A, et al. Colour contrast sensitivity in patients with age-related Bruch's membrane changes. German f Ophthalmol 1995;4:336-41.

41 Spraul CW, Grossniklaus HE. Characteristics of drusen and Bruch's-membrane in postmortem eyes with age-related macula degeneration. Arch Ophthalmol 1998;115:267-73.

42 Young RW. Pathophysiology of age-related macular degeneration. Surv Ophthalmol 1987;31:291-306.

43 Remky A, Elsner AE, Morandi A, et al. Blue-on-yellow perimetry with a SLO: small alterations in the central macula with aging. F Opt Soc Am A Opt Image Sci Vis $2001 ; 18: 1425-36$. 